\title{
Enabling stem cell therapies through synthetic stem cell-niche engineering
}

\author{
Raheem Peerani ${ }^{1}$ and Peter W. Zandstra ${ }^{1,2,3}$
}

\author{
${ }^{1}$ Institute of Biomaterials and Biomedical Engineering, and Department of Chemical Engineering and Applied Chemistry, \\ University of Toronto, Toronto, Ontario, Canada. ${ }^{2} \mathrm{McEwen}$ Centre for Regenerative Medicine, University Health Network, Toronto, Ontario, Canada. \\ 3 Heart and Stroke Richard Lewar Centre of Excellence, Toronto, Ontario, Canada.
}

\begin{abstract}
Enabling stem cell-targeted therapies requires an understanding of how to create local microenvironments (niches) that stimulate endogenous stem cells or serve as a platform to receive and guide the integration of transplanted stem cells and their derivatives. In vivo, the stem cell niche is a complex and dynamic unit. Although components of the in vivo niche continue to be described for many stem cell systems, how these components interact to modulate stem cell fate is only beginning to be understood. Using the HSC niche as a model, we discuss here microscale engineering strategies capable of systematically examining and reconstructing individual niche components. Synthetic stem cell-niche engineering may form a new foundation for regenerative therapies.
\end{abstract}

\section{Introduction}

Stem cell-targeted therapies can be defined as therapies that either use small molecules to regulate endogenous stem cell proliferation and differentiation or involve transplanting autologous and allogeneic stem cells or their in vitro-generated derivatives to enable tissue repair. Preclinical and clinical research based on stem cell-targeted therapies is underway for many diseases, including cancers, heart failure, neural degenerative diseases, autoimmune disorders, and diabetes $(1,2)$. A key aspect in the enabling of these therapies will be the ability to manipulate stem cell interactions with their local microenvironment (a setting known as the niche).

How the stem cell niche, which acts to filter and interpret a wide range of cellular scale physical and biological signals, acts to regulate tissue regeneration based on physiological demand and pathological state remains incompletely understood (3). Clinically, it is evident that the etiologies and pathophysiologies of many diseases, including leukemia, gliomas, Crohn disease, and epithelial skin cancer, involve microenvironmental alterations that perturb stem cell behavior (4).

Key challenges in developing therapies that focus on stem cellextrinsic parameters include the need for a better understanding of the regulatory role of different stem cell-niche components and the development of systems-based approaches to target control points in these complex multidimensional structures. Each of these goals will benefit from bioengineering strategies to synthesize and mimic stem cell-niche components, both in isolation and as an integrated unit. Several excellent reviews have discussed the biology of the stem cell niche, including its various components and their significance (5-7). Herein, we focus primarily on the functional ontology of stem cell-niche components and discuss how bioengineering technologies can be used to recreate these components, both to provide novel insight into the control of stem cell fate and to enable new, clinically relevant strategies for tissue regeneration.

In vivo, stem cell niches create specialized microenvironments, consisting of soluble and surface-bound signaling factors, cell-cell contacts, stem cell niche support cells, ECM, and local mechanical microenvironments (Figure 1). These components are combined with systemic $(8,9)$ and neural $(10,11)$ inputs that either

Conflict of interest: The authors have declared that no conflict of interest exists. Citation for this article: J. Clin. Invest. 120:60-70 (2010). doi:10.1172/JCI41158. directly or indirectly regulate physicochemical cues, such as oxygen, metabolites, and hormones. The stem cell niche, although often represented as a static microenvironment into which stem and progenitor cells are nested, is in fact spatially and temporally dynamic, actively integrating long-term developmental signals with short-term cyclical and injury-mediated regenerative responses (12). Prospective alteration of the properties of stem cell niches may provide new therapeutic strategies by interrupting disease processes or accelerating regenerative healing.

\section{A brief overview of the dynamic HSC niche}

Analysis of the HSC and its microenvironment provides a basis for understanding the design principles and regulatory complexity of a stem cell niche. The HSC niche is used as a prototypical stem cell niche because much is known about interactions between HSCs and their niches. Importantly, the principles underlying the regulation of the HSC niche are broadly applicable to other stem cell niches, although the identity of the particular molecular players may be different. As is true for other stem cell niches, the location, composition, and configuration of the HSC niche changes throughout ontogeny (Figure 2A). Several lines of evidence, including ex vivo embryo cell culture (13), cell lineage-tracking studies $(14,15)$, and analysis of circulating cells in mutant mice (16), suggest that microenvironments for the de novo emergence and maturation of hematopoietic progenitor cells can exist in several locations in the mammalian embryo. Although it is clear that extraembryonic tissue, such as the yolk sac and the placenta, gives rise to early hematopoietic progenitors, the intrinsic and extrinsic signals required for developmental progression into cells capable of fulfilling the functional requirements of definitive HSCs are still poorly understood. Progenitors isolated from the yolk sac and injected transplacentally or into newborn mice become capable of making long-term, multipotent contributions to the hematopoietic system (13) and direct lineage relationships can be traced between progenitors in the yolk sac and adult HSCs; this suggests that sequential in vivo microenvironments (maturation steps) may be necessary for generating cells capable of stable long-term hematopoiesis. Indeed, the failure of conventional tissue culture systems to mimic these developmentally specified microenvironments may account for the fact that upon in vitro differentiation, pluripotent stem cells 


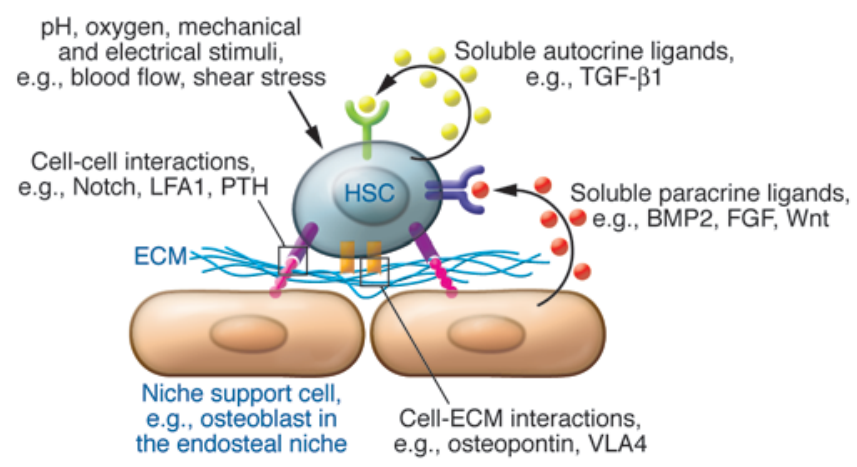

Figure 1

The components of the HSC niche. As a paradigm, a niche can be compartmentalized into signaling pathways activated by soluble cytokines either secreted as autocrine ligands by stem cells themselves or in a paracrine manner by niche support cells, cell-ECM/cell-substrate interactions, cell-cell contacts, mechanical forces, and systemic hormones and physicochemical cues. Examples are given here for components of the HSC niche. LFA1, lymphocyte function-associated antigen-1; PTH, parathyroid hormone; VLA4, very late antigen-4.

(PSCs), including induced PSCs, typically yield progenitors that resemble those found in the yolk sac. It may be necessary to more closely mimic the physical and molecular dynamics of developmental stem cell niches to produce and maintain functional and mature PSC-derived HSCs for cell therapy (3).

Even after definitive transplantable HSCs emerge within the embryo, their anatomical migrations are just beginning. Aortagonad-mesonephros (AGM) resident HSCs start to colonize the fetal mouse liver 10 days post coitum $(17,18)$. Transplantable HSCs in the fetal liver appear to be distinct from adult HSCs in gene expression and functional properties, such as cell cycling and cellular output (19). Whether these differences are associated with the distinct microenvironments, and thus can be mimicked or manipulated using stem cell-niche engineering, or are part of the intrinsic developmental program of HSCs remains to be elucidated.

HSCs can be found in at least two locations within the adult bone marrow: a location known as the osteoblastic niche, near the surface of the endosteum (the cellular lining separating bone from bone marrow that is comprised of different cell types, including osteoblasts, osteoclasts, and stromal fibroblasts), and a location known as the perivascular niche, which is associated with the vasculature (Figure 2B; reviewed in ref. 7). Osteoblasts have been identified as key players in the endosteal niche (reviewed in refs. $20,21)$. Mice with a conditional deletion of the bone morphogenetic protein receptor, type IA (Bmprla) gene have been shown to develop ectopic regions resembling trabecular bone, and the number of these areas correlated with an increase in both the number

\section{Figure 2}

Dynamic interactions between HSCs and the niche. In both the developing embryo (A) and the adult (B), HSC niches vary in time, location, and composition. The HSCs and their interactions with their current niche may provide the required signals to induce de novo hematopoiesis, maturation, or migration into the subsequent niche. In the adult, evidence suggests that HSCs reside in both endosteal and vascular niches that provide different molecular cues to regulate HSC quiescence and response to injury. There is still considerable controversy about the composition and role(s) of niche components and regulatory mechanisms active in the adult and embryonic HSC niches. of osteoblasts and HSCs (22). Similarly, transgenic mice expressing constitutively active parathyroid hormone/parathyroid hormone-related peptide receptor have been found to have increased numbers of osteoblasts and increased numbers of primitive blood progenitors, in part as a result of local increased Notch 1 activation (23). In vivo phenotypic analysis of putative HSCs and new genetic models have contributed to the recent identification of other HSC-associated regulatory axes within the bone marrow (reviewed in refs. 24, 25). In particular, reticular and endothelial cells, major components of the bone marrow vascular microenvironment, appear to be important participants in both the vascular and endosteal stem cell niches (26). How these dynamic and interactive niches integrate local signals to regulate HSC fate remains an important question (27). For example, although recent studies suggest that interactions between HSCs and VEGFR2 are required for hematopoietic engraftment and reconstitution of the vascular niche following myeloablative treatments (28), the role of damage to the bone marrow microvasculature and the associated crosstalk between HSCs and soluble and surface-bound factors in the regenerative process is not well understood. Recreating microenvironments capable of isolating key stem cell-niche parameters and evaluating their impact on defined hematopoietic stem/progenitor cell populations (29) should yield mechanistic insight and new strategies to control cell fate for therapy.

\section{Engineering the stem cell niche}

Establishing the functional equivalents of the stem cell niche. The motivation that drives stem cell-niche engineering is the question of how the extrinsic variables governing the local microenvironment of a stem cell can be recreated in a controlled reproducible manner. As in the preceding section, we primarily use the HSC system to demonstrate the key properties and concepts that should underlie cre-

\section{A Embryonic hematopoiesis}

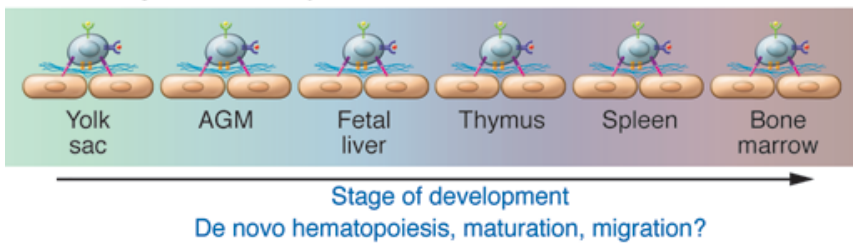

\section{B Adult}

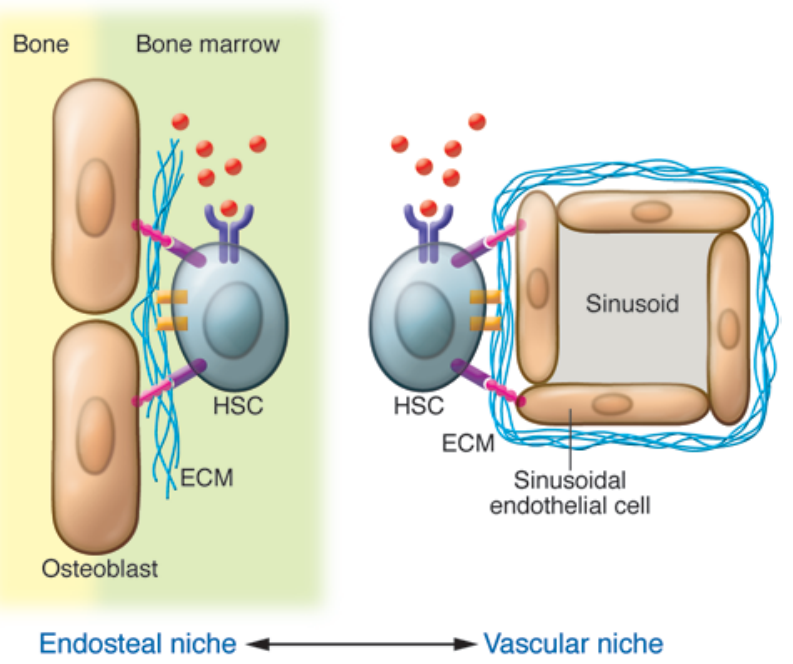




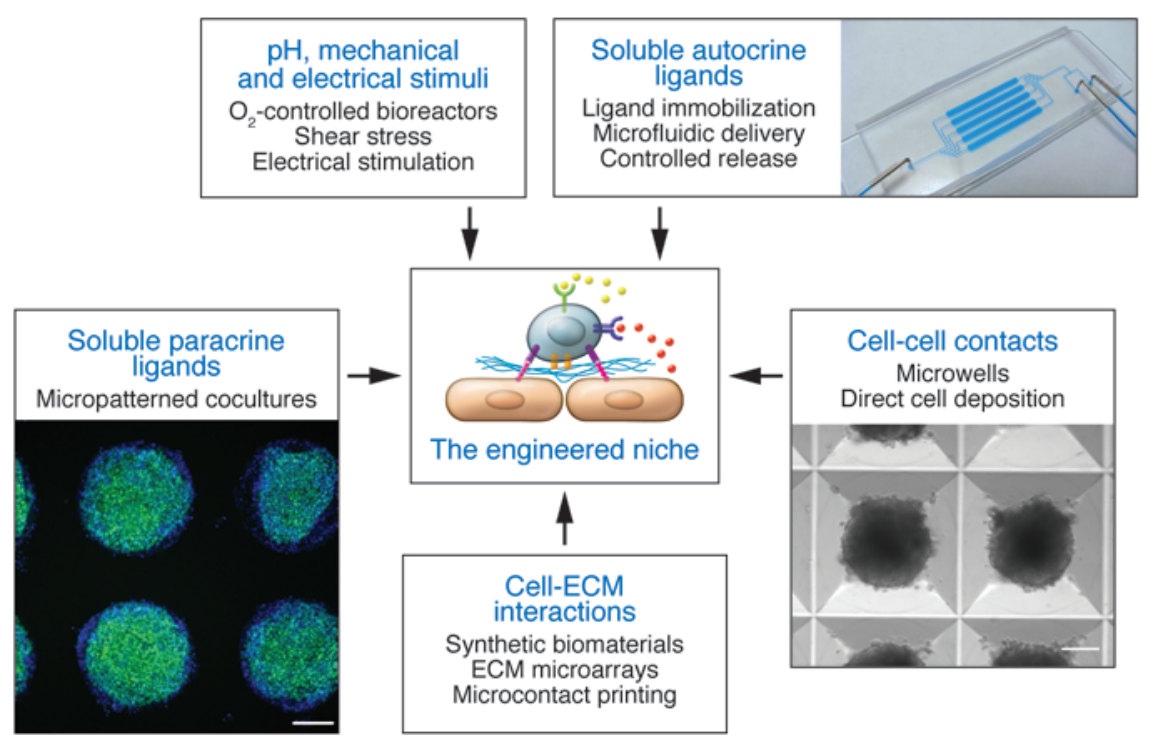

\section{Figure 3}

Strategies to engineer a stem cell niche. There are a variety of approaches to engineer and control individual niche components. These strategies can be multiplexed to produce hybrid devices that simultaneously provide macroscopic (e.g., $\mathrm{O}_{2}$-controlled bioreactors) and microscopic (e.g., micropatterned cocultures) control over the niche and stem cell fate. A poly(dimethyl siloxane) microfluidic device is shown (top right). hES cells were formed into 2,000-cell aggregates in 400- $\mu \mathrm{m}$ microwells (bottom right; scale bar: $100 \mu \mathrm{m}$ ). hES cells were immunostained with Hoechst 33342 (blue) and Oct-4 (green) on 400- $\mu \mathrm{m}$ Matrigel spots (bottom left; scale bar: $200 \mu \mathrm{m}$ ). ating synthetic niches for any stem cell type. Where HSC-specific examples cannot be found to illustrate a particular engineering strategy other stem or non-stem cell examples are provided.

Integral to niche engineering is dynamic control over soluble and surface-bound cytokines, ECM, cell-cell interactions, mechanical forces, and physicochemical cues (Figure 3). The roles of these individual stem cell-niche components are highlighted in Table 1, and example molecules and systems fulfilling these parameters in the context of the hematopoietic system are provided in Table 2. For instance, cell adhesion molecules allow for the localization and homing of HSCs to the niche, whereas ECM provides niche structural support and a stem cell attachment substrate. Using stem cell-niche engineering to study and control stem cell fate has several advantages. First, it can be used as a system to culture cells under more tightly regulated parameters at the cellular level than can be achieved in randomly organized bulk cultures, thereby removing heterogeneity and revealing biological mechanism (30). In this regard, stem cell-niche engineering is a technical tool to control cell signaling and fate. Second, it can be used as a means to determine the relative contributions of different niche components to the regulation of stem cell fate. For instance, the effect of cell-cell contact can be uncoupled from the effects of soluble ligand-based activation of signaling pathways by controlling the geometry of a culture at the single cell level (31). High-throughput approaches are also available to screen niche parameters in a combinatorial manner $(32,33)$. Finally, stem cell-niche engineering may reveal novel strategies to modulate stem cell niches in vivo for clinical benefit; for example, it may be possible to specifically disrupt interactions between stem cells and their niche (See Future directions: clinical translation of engineered niches below) or develop a way to guide the integration and functional maturation of cells into injured tissue (34). In the following sections, we discuss how various microscale technologies will enable stem cell-niche engineering at the molecular and cellular scale.

Engineering immobilized cytokine presentation. Localized growth factor delivery permits precise control over their concentrations and gradients and enables context-dependent signaling (Table 1). Ligand immobilization on surfaces represents one means of localized delivery and can involve tethering cytokines to surfaces using covalent chemical modification (35), incorporating ligands into biomaterial scaffolds (36), or simple protein adsorption (37). Within the hematopoietic system, it has been shown that a fusion protein combining SCF with a cellulose-binding domain is capable of inducing ligand-dependent receptor polarization on hematopoietic progenitor cell membranes, albeit with altered downstream signaling kinetics relative to similarly supplemented soluble SCF (38). This work and similar studies in other stem cell systems $(39,40)$ has demonstrated that the mode of cytokine presentation can impact downstream signaling fluxes and thus stem and progenitor cell fate. Localized cytokine presentation, which mimics in vivo mechanisms of cytokine delivery, appears to allow for a larger range of stem cell fate options than soluble factor delivery. For example, the Notch ligand Delta1, as part of a Delta1-Fc chimeric protein, appears more effective at expanding transplantable human HSC numbers from cord blood CD $133^{+}$ cells when presented as an immobilized growth factor than when soluble (41). While not presently used in stem cell cultures, there is considerable opportunity to use spatial gradients in ligand presentation to control cell homing, adhesion, spreading, and migration. For instance, nerve growth factor patterning in three dimensions guides neuronal migration (42), and controlled surface deposition of matrix proteins and growth factors controls cell spreading (43). In two dimensions, microcontact printing $(\mu \mathrm{CP})$ and microfluidic devices (see Sidebar 1 for a more detailed explanation of these approaches) have been used to directly pattern protein gradients onto surfaces $(44,45)$.

Importantly, although seemingly complex, these technologies are quite accessible; for example, high-throughput strategies to present combinations of ligands to stem cells have been achieved using commercially available microarray equipment. These microarrays consist of combinations of immobilized ligand and ECM spotted onto a two-dimensional substrate. In one study Wnt, Notch, and bone morphogenetic protein 2 (BMP2) were deposited as an array on slides to demonstrate how interactions between different combinations of matrix proteins and cytokines can modulate neural stem cell self-renewal and differentiation (33). To summarize, the use of the various approaches to ligand presentation described in this section is to guide cellular behavior at the microscale, with 


\section{Table 1}

Functional ontogeny of stem cell-niche components

\section{Niche component}

Soluble and surface-bound cytokines

Cell-ECM interactions

Cell-cell interactions

Mechanical forces

Physicochemical cues

Systemic factors

\section{Functional role}

Mediate localized signaling

Fine tune gradients and cytokine-activation thresholds

Allow for context dependent signals

Regulate retention and mobilization of cells

Provide structural integrity to the niche

Allow for cell polarization and matrix mediated signaling

Provide a substrate for cell migration

Provide structural support

Retain cells in the niche

Regulate niche size

Regulate stem cell mobilization

Structural support

Juxtacrine signaling

Allow for gradients and positional information

Allow for autoregulatory control of niche size

Allow niche to respond to external physical forces and cues

Regulate signaling and transcription via stress responsive networks

Allow niche to respond to external physical forces and chemical cues

Link niche to metabolic cues and homeostatic demands

Regulate nutritional status of the local microenvironment

Link niche to metabolic cues and homeostatic demands

See Table 2 for examples of molecules and systems related to these niche components.

increased precision and homogeneity relative to bulk cultures. Biologically this allows for precise control over the concentration of the signal and the establishment of signaling gradients on the cellular scale. Clinically, such approaches could be used to expand stem cells ex vivo or allow for greater functionality of scaffolds that can be cotransplanted with cells.

Engineering soluble and dynamic cytokine presentation. The controlled delivery of ligands using biodegradable scaffolds or microfluidic devices provides growth factors in a temporally and spatially restricted fashion. The kinetics of ligand release can be engineered using programmed material degradation, as has been demonstrated with controlled release of dexamethasone to induce osteoblastic differentiation of human mesenchymal stem cells, using photopolymerization to attach dexamethasone to a gel with hydrolyzable lactide bond (46). Cellular architecture and matrix infiltration can also be dynamically controlled by enzyme-based material degradation (47).

Microfluidic devices are capable of providing linear (48) and logarithmic gradients (49) of ligand to cells by manipulating flow rates and flow profiles in the cellular environment. Combinations of cytokine gradients can be applied, as demonstrated for EGF, FGF, and PDGF in a study that examined astrocyte differentiation from human neural stem cells (50). This study suggested that it might be possible to mimic in vivo growth factor concentrations and thresholds for signaling and differentiation in vitro. Owing to high functionality and small sample requirements, microfluidic systems provide excellent opportunities for applications requiring high-throughput cell culture, screening, and analysis (51). For stem cells, this may include screening for self-renewal, differentiation, and antiapoptosis factors as well as those affecting aggregation, spreading, and attachment.
Engineering stem cell-ECM interactions. Stem cell-ECM interactions are important in the regulation of several stem cell-niche functions, including homing, mobilization, structural support, and signals for self-renewal and differentiation. Approaches to regulate stem cell-ECM interactions include synthesizing novel biomaterials for stem cell culture (52), fabricating scaffolds in three dimensions with microscale or nanoscale topography (53), micropatterning ECM in two dimensions (54), and high-throughput ECM microarrays. ECM microarrays are particularly useful for the design of novel surface coatings to grow stem cells on and for high-throughput interrogation of the role of matrix proteins in controlling stem cell fate (55). Synthetic biomaterials and recombinant protein forms of ECM have been used for stem cell maintenance, differentiation, and in vivo transplantation $(52,56)$. For example, increased numbers of hematopoietic progenitors and substantial differences in gene expression were observed when bone marrow isolates were cultured on collagen I versus control suspension cultures (57). In addition, aminated nanofiber scaffolds have been used to expand $\mathrm{CD} 34^{+} \mathrm{CD} 45^{+}$mouse HSCs (58).

High-throughput ECM microarrays offer the ability to screen cell-ECM interactions with greater efficiency compared with conventional approaches, such as coating a typical microplate with a biopolymer. A robotic microarrayer has been created to generate a polymer array capable of analyzing 1,700 cell-biomaterial interactions simultaneously (55). It has been used to screen interactions between human ES (hES) cells and numerous biomaterials, with hits being defined as those maintaining hES cell self-renewal. Commercial microarrays have also been used to interrogate mouse ES cell differentiation into hepatocytes on combinations of laminin, fibronectin, collagen I, collagen II, and collagen IV (59). In combination, these studies suggest that microarray tech- 
Table 2

Example molecules and systems in association with the adult HSC-niche components

Examples of HSC niche- $\quad$ Representative biological activity
associated molecules

Reference

\section{Soluble and surface-bound cytokines}

Angiopoietin

CXCL12

$\mathrm{SHH}$

SCF

WNT

DKK1

FGF

\section{BMP}

Calcium ions

\section{Cell-ECM interactions}

$\beta_{1}$ integrins

Osteopontin

VLA4

Collagen I

Cell-cell interactions

LFA1

Annexin-II

PTH

Notch

\section{Mechanical forces}

Shear-stress/blood flow

ECM structure

Physicochemical cues

Hypoxic microenvironments

ROS

\section{Systemic factors}

Neural input

Hormonal input
Regulates quiescence and maintains in vivo long-term repopulating activity of HSCs 97

Retains HSCs in the bone marrow; induces HSC transendothelial migration Induces proliferation of primitive human hematopoietic cells via BMP signaling Increases HSC survival in vitro; contributes to the self renewal and maintenance of HSCs in vivo; gradients of SCF may allow for HSCs to home to the marrow niche Induces the proliferation and expansion of the HSC pool Antagonizes Wnt signaling thereby promoting HSC quiescence An FGF gradient between the vascular niche (high) and osteoblastic niche (low) has been suggested to regulate $\mathrm{HSC}$ recruitment Promotes HSC maintenance through negative control of the size of the endosteal niche Promote adhesion of HSCs to collagen I in the niche

Highly expressed on blood forming HSCs but may not directly relate to HSC activity 123 Matrix glycoprotein thought to limit HSC number $\quad 124$ Monoclonal neutralizing antibody specific for VLA4 induces HSC mobilization $\quad 125$ Promotes HSC Proliferation

Promotes HSC adhesion to osteoblasts $\quad 126$ Promotes homing to the niche 127 Regulates osteoblast proliferation and therefore the number of HSCs in the niche (niche size) 23 Regulates HSC number via osteoblasts

Increases number of ESC-derived hematopoietic progenitors in vitro and in the AGM region in vivo via nitric oxide signaling HSCs cultured in smaller microcavities have decreased DNA synthesis and higher levels of HSC marker expression in vitro

Increase number of hematopoietic progenitors in vitro and in vivo via competitive interactions between a soluble VEGFR1 and VEGFR2 for VEGF

Mediate N-cadherin expression to retain HSCs in the niche

DKK1, Dikkopf-1; LFA1, lymphocyte function-associated antigen-1; PTH, parathyroid hormone; SHH, Sonic hedgehog; VLA4, very late antigen-4.

niques will allow the interrogation of stem cell-substrate interactions quickly in order to create better in vitro niches for stem cells and will provide us with an in depth understanding of the role of matrix-derived signals in stem cell fate.

Engineering cell-cell interactions. Cell-cell contact appears to be of particular importance for stem cells such as HSCs, as they form heterogeneous cell contacts with osteoblasts, adipocytes, and megakaryocytes in the endosteal niche (Table 2). To mimic T cell interactions with macrophages in vitro, HSCs have been cultured with magnetic beads conjugated to the Notch ligand delta-like ligand 4 (DLL4) to induce their differentiation into T cells (60). Furthermore, direct coculture of umbilical cord-derived HSCs with the OP9 stromal cell line engineered to express DLL1 has been shown to expand HSCs with the ability to engraft the thymus of immunodeficient mice (61). Another approach to engineering cellcell contacts has been to tether proteins that are typically found at the cell surface to biomaterials. Coating surfaces with immobilized E-cadherin fused with the Fc portion of IgG demonstrated that homotypic interactions between mouse ES cells can be largely replaced by E-cadherin presentation (62); if this result translates to hES cells, it may enable increased efficiency of single hES cell cloning. Cell-cell contact can also be controlled by the direct deposition of cells onto surfaces (63). As an example, mouse ES cells have been immobilized onto surfaces in clusters or as single cells to demonstrate that homotypic ES cell interactions depress colony-forming capacity in a manner associated with E-cadherin ligation (64). In addition, bow tie-shaped microwells, designed to limit the contact area between cells, have been used to demonstrate that homotypic ES cell interactions regulate specification toward neuroectoderm (possibly via connexin 43 ligation modulation) (65). In a very elegant non-stem cell example, stromal cells and hepatocytes have been cultured on micromachined silicon substrates with mobile 


\section{Sidebar 1}

\section{Engineering techniques to control individual niche components}

\section{Soft Lithography}

Soft lithography refers to a family of techniques for fabricating elastomeric (soft) stamps and molds. Typically, it is achieved by molding poly(dimethyl siloxane) (PDMS) into a silicon wafer that already has been pattered, using standard photolithography techniques. Microscale to nanoscale features can be incorporated into the design. Each PDMS mold forms a negative replica of the mask (patterned silicon wafer) that can then be used as a stand-alone device or stamp.

\section{Microcontact printing}

Microcontact printing in biological applications is a technique that directly deposits protein, ECM, or cells onto a substrate with stamp that has microscale dimensions. Most commonly, PDMS stamps fabricated using soft lithography are used and "inked" with a protein, ECM, or cell solution. The stamp is then placed into conformal contact with the substrate and the "ink" is transferred to the substrate.

\section{Microfluidic devices}

For biological applications, microfluidic devices are commonly made out of PDMS using soft lithography. The term microfluidic applies to any device that has at least one dimension (e.g., height, channel width, orifice diameter) in the microscale. Devices can be used to seed cells onto a tissue-culture substrate with microscale precision, plus or minus cocktails of cytokines, depending upon the design of the device.

\section{ECM microarrays}

The term extracellular microarrays refers to arrays of ECM proteins or other biomaterials deposited onto a substrate for further cell culture. They differ from DNA microarrays insofar as they use a different material (protein/polymer versus nucleic acid), and ECM microarray fabrication is more sensitive to environmental variables such as temperature and humidity. Devices used to spot DNA microarrays can often be adapted to print ECM microarrays.

parts, in order to dynamically modulate their interactions (66). Applying this approach to stem cell-niche interactions may provide novel insights into the distinct roles of cell contact and paracrine signaling in stem cell-fate decisions.

Microscale engineering of cocultures allows for greater control over interactions between cell types. Conventional cocultures of two or more cell types involve direct coculture, with heterotypic cell-cell contacts and paracrine signaling; trans-well cultures with limited cell-contact; or culture in conditioned media, which isolates soluble signals. In all three of these approaches, inductive cues provided by the cells are imprecisely applied, thereby impairing the ability to discern mechanism(s). In an important illustrative counter example, primary rat hepatocytes have been cocultured with mouse NIH 3 T3 fibroblasts on micropatterned surfaces to investigate the role of homotypic and heterotypic cell-interactions on albumin and urea synthesis (67). Two interesting observations were made. First, maximizing heterotypic interactions between cells was necessary to maintain proper hepatocyte phenotype. Second, homotypic interactions among fibroblasts influenced the ability of fibroblasts to regulate hepatocyte behavior. As another approach, a layer-by-layer deposition using ionic polymers has been used to culture mouse ES cells and fibroblasts with microscale organization in two dimensions (68). Furthermore, dynamic micropatterned cocultures that allow for supporting cells to be added and removed have been developed using parylene-C stencils. In a proof-of-principle experiment, mouse ES cells were sequentially micropatterned with fibroblasts and hepatocytes (69). While not commonly employed for HSC culture at the moment, micropatterned cocultures of HSCs with inductive cell types could provide a novel strategy to discern the effects of paracrine signaling versus heterotypic cell-cell interactions on HSC fate.

Engineering mechanical forces on stem cells. Applying mechanical strain to stem cells has been shown to influence their self-renewal and differentiation capacities (Tables 1 and 2) (70,71). Strain can be applied by mechanical strain-loading devices (70) or by regulating the substrate compliance and cell culture geometry (71). Microscale cantilevers can be used to apply and measure forces at the cellular and subcellular level (72). Recently, biomechanical forces acting on the vascular wall have been implicated in the development of hematopoietic cell progenitors $(73,74)$. Silent heart embryos, which by definition have no heartbeat or circulation, have severely reduced numbers of hematopoietic progenitors, suggesting that shear forces from blood flow may be required for the appropriate development of the hematopoietic system (74). The fluid force appears to act via nitric oxide signaling, as adding exogenous nitric oxide donors partially rescued hematopoietic development. In another study, it was demonstrated that applying shear forces increases the number of ES cell-derived colony-forming cells (73). In other stem cell systems, matrix elasticity appears to specify mesenchymal stem cell fate such that soft, stiff, and rigid matrices give rise to neurogenic, myogenic, and osteogenic cells, respectively (75). Furthermore, cell shape, which can change the physical stress a cell is under, appears to regulate the commitment of mesenchymal progenitor cells via endogenous RhoA activity, such that round cells differentiate into adipocytes, whereas flat, spread cells turn into osteoblasts (76). Forming multicellular aggregates of normal rat kidney epithelial cells on micropatterned surfaces has revealed that tissue geometry can generate regions of localized proliferation in areas of high traction. These regions, which were found at the edges, could be predicted by a finite-element model of multicellular mechanics and were dependent upon an intact actin cytoskeleton and mediated by cadherin-based cell-cell interactions. It is likely that stem cells are also responsive to such distributions in mechanical forces; the application of these tools to synthetic stem cell-niche engineering should prove fruitful. Overall, deconvolution of contributors of mechanically responsive signaling and 
the soluble factor signals that these directly or indirectly modulate (77) will require precise stem cell niche-engineering strategies.

Engineering physicochemical cues: oxygen and $p H$. Adaptive responses to hypoxia are required for development, facilitating oxygen and nutrient delivery to the rapidly growing embryo, and for the normal development and patterning of the cardiovascular system (reviewed in ref. 78). As a regulator of oxygen homeostasis, HIF induces a network of genes related to angiogenesis, erythropoiesis, and glucose metabolism. Hypoxic regions have been identified in the yolk sac mesoderm, in which HIF-1 $\alpha$ and VEGF colocalize to induce blood vessel formation. These observations point to oxygen-regulated activation of growth factor signaling as an important component of hematopoietic niches associated with the emerging embryonic blood islands (areas of hematopoiesis in the AGM region). By encapsulating mouse ES cells in a hydrogel matrix and driving their hematopoietic differentiation under hypoxic conditions in bioreactors, it has been shown that the secreted form of VEGFR1 competes with VEGFR2 to control the extent and timing of VEGF-mediated signaling (79). This in vitro model has since been shown to be predictive of the temporal (stage of development) and microenvironmental (oxygen-dependent) events that occur in vivo during embryonic hematopoietic and endothelial development (80). Likewise, it has been shown that small molecules such as 5-fluorouracil, which is used as an anticancer drug, can interfere with ROS in HSCs (81). This drug suppresses $\mathrm{N}$-cadherin expression, and its application to mouse HSCs is speculated to cause quiescent HSCs to detach from the stem cell niche. This effect was mitigated by administration of a potent antioxidant, $\mathrm{N}$-acetyl cysteine, suggesting that ROS species regulate HSC retention in the stem cell niche.

Recently, a microscale device has been fabricated to regulate oxidative microgradients in culture (82). Using electrodes 3-40 $\mu \mathrm{m}$ in width within gas-permeable microchannels, precise doses of dissolved oxygen were administered via electrolysis. Biological implementation of this device was assessed by monitoring $\mathrm{C} 2 \mathrm{C} 12$ myoblast (a skeletal muscle precursor cell line) apoptosis, due to localized hypoxia and calcium release due to ischemia. Controlling $\mathrm{pH}$ may also be important, as it has been demonstrated that small changes, up or down, in the $\mathrm{pH}$ of the medium provided to ES cells results in an approximately $50 \%$ decrease in the yield of cells capable of forming embryoid bodies (three dimensional spheroids of differentiating ES cells) (83). Ultimately, it is likely that control of oxygen and $\mathrm{pH}$ in artificial stem cell niches will require new sensor technologies, capable of cell-level measurement and control (84), enabling prospectively controllable links between cell signaling, gene regulation, and metabolic networks (85).

Predictions and regulation of network interactions among stem cellniche components. A major limitation of the analysis and reconstruction of the stem cell niche using individual parameters is that the niche is a multicomponent system in vivo. The reason for this conceptual impediment is not due to a lack of insight, rather it is due to the complexity of generating such systems in vitro. From the above descriptions of the stem cell niche, it is clear that stem cell cultures in vivo and in vitro are complex, nonlinear, multiple-input, multiple-output systems, with considerable temporal and spatial heterogeneity. Extracellular interaction networks mediated through the secretion of cytokines and inhibitors among different cell types and intracellular communication pathways mediated through signaling pathway crosstalk require their own methods of measurement and characterization.
With respect to measurement, molecular barcodes - unique tags capable of identifying several individual biological species - are emerging as an important tool for rapidly analyzing large numbers of locally produced biological molecules simultaneously. For instance, researchers have used quantum dot barcodes to measure factors and cells produced under controlled culture conditions (86). Applying such technologies to stem cell cultures will allow us to parse out intercellular communication networks established by the stem cells and their derivatives. Regarding characterization, systems biology approaches to studying interactions between stem cells and their niches, along with in silico modeling, have generated relevant network models capable of predicting whether a stem cell will self-renew or differentiate $(29,87,88)$. These models can provide mechanistic insight into how stem cell-culture heterogeneity influences culture output, can be used to optimize culture output, and can predict how dysregulation may occur in malignancies $(29,89)$. Connecting extracellular signaling events to intercellular signaling and ultimately to changes in transcription and phenotype requires further effort. Pioneering studies connecting the activation of downstream transcription networks that regulate pluripotency in mouse ES cells to signaling cascades induced by factors that control self-renewal, such as leukemia inhibitory factor and BMP $(90,91)$, are an important step in this direction.

Extending stem cell-niche engineering to three dimensions poses additional challenges that once overcome can provide a closer mimicry of the in vivo microenvironment $(92,93)$. Controlling tissue morphogenesis is a feasible task in three dimensions but requires a scaffold that regulates the presentation of ligand, is sensitive to stimuli, provides structural support, and has the ability to induce cell migration or invasion into the scaffold (92). Additional complexity in the fabrication of three-dimensional stem cell niches may also be achieved by introducing either microfluidic (94) or photochemical (95) control over the generation of gradients in the scaffold. The use of such scaffolds in vivo is just beginning to be explored as a means to deliver stem cells or their derivatives; however, they provide an opportunity to use stem cell-niche engineering within a living organism (96).

\section{Future directions: clinical translation of engineered niches}

A link between stem cells and certain cancers has been postulated through the cancer stem cell hypothesis, which supposes that tumor-initiating cells (CICs), those cells capable of reconstituting a tumor upon serial transplantation into another organism, descend either from stem cells or from their differentiated progeny that have acquired stem cell-like characteristics. Thus, the bulk of a tumor may consist of non-CICs, with only a subset of cells being required to propagate it. This hypothesis, if true, has implications for drug therapy, such that strategies that solely reduce the bulk of a tumor through the suppression of proliferation may reduce tumor size without removing the quiescent CICs. An ongoing area of research is how the physiological stem cell niche itself is modulated in cancer. Although it is clear that (stem) cell-intrinsic mutations are fundamental to tumor initiation, progression, and metastasis, the role of the normal or abnormal stem cell niche in regulating cell responses to these mutations is unknown and presents an open-ended question. The targeting of CIC-niche interactions is emerging as a promising area of therapy. Targeting the stem cell niche to support 


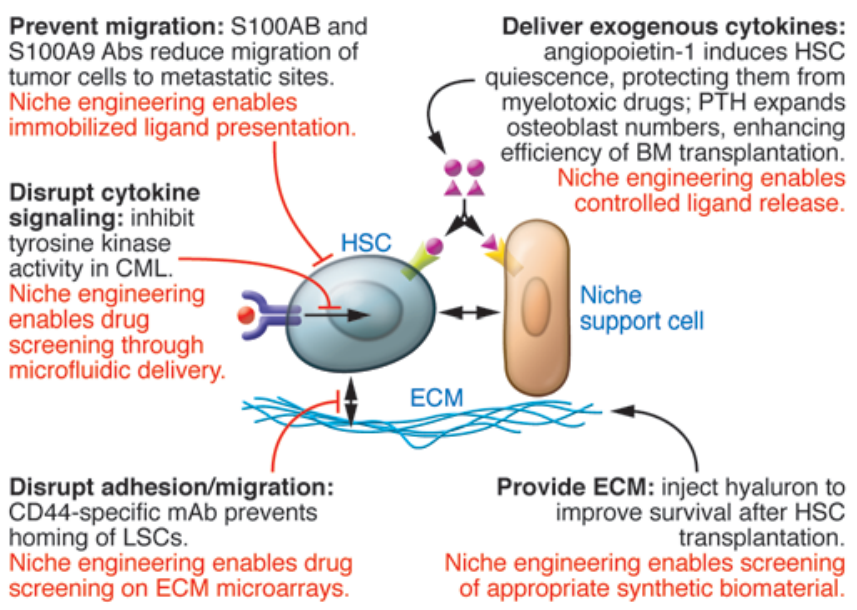

\section{Figure 4}

Engineering and modulating the niche in vivo. This figure provides an overview of different clinically relevant strategies that may be used to manipulate the in vivo stem cell niche. Examples provided illustrate the molecular targeting of particular stem cell-niche interactions. CML, chronic myeloid leukemia; LSC, leukemic stem cell.

endogenous stem cell survival during chemotherapy and/or to enhance hematopoiesis after chemotherapy is also a possibility.

Candidate approaches to target interactions between the stem cell niche and either CICs or endogenous HSCs include the modulation of cytokine signaling, cell adhesion molecules, homing mechanisms, and niche support cells to regulate stem cell fate, position, and cell cycle status (Figure 4). For example, injection of mice with angiopoetin-1, the ligand for the Tie- 2 receptor, induces HSC quiescence, thereby protecting them from myelotoxic drugs and allowing for greater survival after chemotherapy (97). In addition, knowledge that osteoblasts and parathyroid hormone are critical in the endosteal HSC niche has been used to develop ways to expand the number of osteoblasts, and consequently the number of resident HSCs capable of being mobilized to the peripheral circulation, and to increase the number of HSCs homing to the stem cell niche using standard bone marrow transplantation protocols (23). These treatments may provide ways to counteract the myelotoxic effects of chemotherapy.

Targeting stem cell adhesion and homing has been successful in slowing leukemic progression in animal models. For instance, treating mice with hyaluronic acid (an extracellular component of the vascular HSC niche) after 5-fluorouracil administration improves the hematopoiesis-supportive function of bone marrow accessory cells, resulting in increased hematopoietic recovery and survival (98). In addition, neutralizing CD44 on so-called leukemic stem cells in mice with acute myeloid leukemia (AML) prevents them homing to the niche, thus preventing their self-renewal and maintenance $(99,100)$. Furthermore, using neutralizing antibodies specific for integrin $\alpha_{4} \beta_{1}$ (also known as very late antigen-4 [VLA4]) in conjunction with cytarabine chemotherapy prevents the development of AML in a SCID xenograft transplantation model, by preventing the attachment of AML leukemic stem cells to fibronectin in the niche (101). Proteasome inhibitors have also been used to inhibit the migration of AML blasts to stromal cell-derived CXCL12 (102). These inhibitors have a dual effect, overcoming conventional drug resistance by directly inducing tumor cell cytotoxicity and inhibiting adhesion of multiple myeloma cells to the bone marrow. Furthermore, the CXCR4 antagonist AMD3465 has been shown to increase sensitivity to chemotherapy in promyelocytic leukemia after treatment with another drug (AMD3100) involved with normal HSC mobilization (103).

Targeting the "premetastatic" niche, an environment thought to recruit hematopoietic and endothelial precursors to a site of future metastasis before tumor cell invasion, has been achieved in some model systems (104). Formation of the premetastatic niche can be prevented in lung cancer via administration of a neutralizing VEGFR1-specific antibody (105). Likewise, antibodies specific for chemoattractants reduced migration of CICs to metastatic sites (106). Together, these examples demonstrate that several components of the niche lend themselves to therapeutic intervention in order to suppress tumor initiation and metastasis.

The potential uses of engineered stem cell niches in expanding the therapeutic options available for treating diseases are manifold. First, one can screen for new drug candidates that specifically target interactions between stem cells and their niche. For instance, high-throughput microenvironment microarrays have been used to identify 192 unique ECM combinations that trigger the differentiation of bipotential human mammary progenitor cells and control their ability to acquire certain molecular characteristics of cancer $(107,108)$. Using such combinatorial arrays in conjunction with small molecule screens and drug libraries will aid the drug discovery process and possibly identify factors that minimize malignant transformation and metastasis. Second, stem cell-niche engineering can be used to generate uniform cell populations from PSCs in vitro. These derivatives can then be used in direct transplantation (109). Several cell culture systems have been employed to improve homogeneity and direct the differentiation of stem cells into various cell types using microfabricated platforms $(110,111)$. Moving toward hybrid cell culture systems that use both microscale approaches to present ligand, ECM, and mechanical forces at the cellular level and macroscopic approaches, such as bioreactors (79) to control shear stress (112) and regulate oxygen tension $(113,114)$, should prove fruitful in cell therapy manufacturing. Third, targeting the in vivo stem cell niche for therapy beyond using drugs and small molecules may be possible by using microengineered substrates that act as artificial niches to which endogenous stem cells can home and attach. For instance, endogenous neural stem cells can migrate and graft onto laminin-coated substrates in vitro, and such engrafted scaffolds could be placed at the site of a spinal cord injury to help promote regeneration (115). Likewise, endothelial cells grafted onto synthetic materials before implantation have been shown to increase de novo blood vessel formation and density (116). The tools of stem cell-niche engineering may enable novel, minimally invasive strategies to treat disease.

\section{Summary}

The molecular mechanisms that govern stem cell fate are complex. Extrinsic control over these processes will require the correct macroscopic and microscopic cues to elicit desired behavior. Using a stem cell-niche paradigm to understand stem cell biology allows the complexity of the system to be broken down into individual components, including soluble signals, cell-substrate interactions, and cell-cell contacts. Several microscale approaches to regulate each of these variables have been employed, includ- 
ing ligand immobilization, surface patterning, ECM microarrays, and dynamic microscale cocultures. Multiplexed systems using a combination of these approaches are starting to emerge, with a greater number of basic scientists and clinical researchers taking an interest in moving the field toward therapies. In general, microscale technologies that regulate stem cell-niche parameters have reached the proof-of-principle stage, where a particular technique is shown to regulate stem cell behavior without deep mechanistic insight into how this approach regulates fate at the molecular level. Demonstrating how microscale approaches activate signaling pathways and modulate specific downstream transcription factors represents an important next step toward microenvironment-mediated cell fate (re)programming. The comprehensive exploration and exploitation of the stem cell niche and its components is an important aspect in using stem cells in regenerative medicine.

\section{Acknowledgments}

This work is funded by the Canadian Institutes of Health Research (CIHR) (MOP-57885), the Natural Sciences and Engineering Research Council (NSERC) of Canada, and the Canadian Stem Cell Network. We would like to thank Mark Ungrin, Geoff Clarke, Elizabeth Csaszar, and Dan Kirouac for critical review and comments on this manuscript. Images of microwells and microfluidic devices were provided by Mark Ungrin and Faisal Moledina, respectively. R. Peerani is a recipient of an NSERC Postgraduate Doctoral Scholarship (PGS D). P.W. Zandstra is the Canada Research Chair in Stem Cell Bioengineering.

Address correspondence to: Peter W. Zandstra, TD-CCBR Rm. 1116, 160 College Street, University of Toronto, Toronto, Ontario, Canada M5S 3E1. Phone: (416) 978-8888; Fax: (416) 978-2666; E-mail: Peter.zandstra@utoronto.ca.
1. Gersh BJ, Simari RD, Behfar A, Terzic CM, Terzic A. Cardiac cell repair therapy: a clinical perspective. Mayo Clin Proc. 2009;84(10):876-892.

2. Trounson A. New perspectives in human stem cell therapeutic research. BMC Med. 2009;7:29.

3. Discher DE, Mooney DJ, Zandstra PW. Growth factors, matrices, and forces combine and control stem cells. Science. 2009;324(5935):1673-1677.

4. Lane SW, Scadden DT, Gilliland DG. The leukemic stem cell niche: current concepts and therapeutic opportunities. Blood. 2009;114(6):1150-1157.

5. Jones DL, Wagers AJ. No place like home: anatomy and function of the stem cell niche. Nat Rev Mol Cell Biol. 2008;9(1):11-21.

6. Scadden DT. The stem-cell niche as an entity of action. Nature. 2006;441(7097):1075-1079.

7. Kiel MJ, Morrison SJ. Uncertainty in the niches that maintain haematopoietic stem cells. Nat Rev Immunol. 2008;8(4):290-301.

8. Carlson ME, Hsu M, Conboy IM. Imbalance between pSmad3 and Notch induces CDK inhibitors in old muscle stem cells. Nature. 2008;454(7203):528-532.

9. Conboy IM, Conboy MJ, Wagers AJ, Girma ER, Weissman IL, Rando TA. Rejuvenation of aged progenitor cells by exposure to a young systemic environment. Nature. 2005;433(7027):760-764.

10. Spiegel A, Kalinkovich A, Shivtiel S, Kollet O, Lapidot T. Stem cell regulation via dynamic interactions of the nervous and immune systems with the microenvironment. Cell Stem Cell. 2008;3(5):484-492.

11. Katayama Y, et al. Signals from the sympathetic nervous system regulate hematopoietic stem cell egress from bone marrow. Cell. 2006;124(2):407-421.

12. Johnston LA. Competitive interactions between cells: death, growth, and geography. Science. 2009;324(5935):1679-1682.

13. Yoder MC, Hiatt K, Dutt P, Mukherjee P, Bodine DM, Orlic D. Characterization of definitive lymphohematopoietic stem cells in the day 9 murine yolk sac. Immunity. 1997;7(3):335-344.

14. Zovein AC, et al. Fate tracing reveals the endothelial origin of hematopoietic stem cells. Cell Stem Cell. 2008;3(6):625-636.

15. Samokhvalov IM, Samokhvalova NI, Nishikawa S. Cell tracing shows the contribution of the yolk sac to adult haematopoiesis. Nature. 2007;446(7139):1056-1061.

16. Rhodes KE, et al. The emergence of hematopoietic stem cells is initiated in the placental vasculature in the absence of circulation. Cell Stem Cell. 2008;2(3):252-263.

17. Houssaint E. Differentiation of the mouse hepatic primordium. II. Extrinsic origin of the haemopoietic cell line. Cell Differ. 1981;10(5):243-252.

18. Cumano A, Godin I. Ontogeny of the hematopoi- etic system. Annu Rev Immunol. 2007;25:745-785.

19. Bowie MB, et al. Identification of a new intrinsically timed developmental checkpoint that reprograms key hematopoietic stem cell properties. Proc Natl Acad Sci U S A. 2007;104(14):5878-5882.

20. Wu JY, Scadden DT, Kronenberg HM. Role of the osteoblast lineage in the bone marrow hematopoietic niches. J Bone Miner Res. 2009;24(5):759-764.

21. Garrett RW, Emerson SG. The role of parathyroid hormone and insulin-like growth factors in hematopoietic niches: physiology and pharmacology. Mol Cell Endocrinol. 2008;288(1-2):6-10.

22. Zhang J, et al. Identification of the haematopoietic stem cell niche and control of the niche size. Nature. 2003;425(6960):836-841.

23. Calvi LM, et al. Osteoblastic cells regulate the haematopoietic stem cell niche. Nature. 2003;425(6960):841-846.

24. Garrett RW, Emerson SG. Bone and blood vessels: the hard and the soft of hematopoietic stem cell niches. Cell Stem Cell. 2009;4(6):503-506.

25. Kiel MJ, Morrison SJ. Maintaining hematopoietic stem cells in the vascular niche. Immunity. 2006;25(6):862-864.

26. Xie Y, et al. Detection of functional haematopoietic stem cell niche using real-time imaging. Nature. 2009;457(7225):97-101.

27. Lo Celso C, et al. Live-animal tracking of individual haematopoietic stem/progenitor cells in their niche. Nature. 2009;457(7225):92-96.

28. Hooper AT, et al. Engraftment and reconstitution of hematopoiesis is dependent on VEGFR2-mediated regeneration of sinusoidal endothelial cells. Cell Stem Cell. 2009;4(3):263-274.

29. Kirouac DC, Madlambayan GJ, Yu M, Sykes EA, Ito C, Zandstra PW. Cell-cell interaction networks regulate blood stem and progenitor cell fate. $\mathrm{Mol}$ Syst Biol. 2009;5:293.

30. Peerani R, et al. Niche-mediated control of human embryonic stem cell self-renewal and differentiation. EMBO J. 2007;26(22):4744-4755.

31. Nelson CM, Chen CS. Cell-cell signaling by direct contact increases cell proliferation via a PI3K-dependent signal. FEBS Lett. 2002;514(2-3):238-242.

32. Flaim CJ, Teng D, Chien S, Bhatia SN. Combinatorial signaling microenvironments for studying stem cell fate. Stem Cells Dev. 2008;17(1):29-39.

33. Soen Y, Mori A, Palmer TD, Brown PO. Exploring the regulation of human neural precursor cell differentiation using arrays of signaling microenvironments. Mol Syst Biol. 2006;2:37.

34. Song $\mathrm{H}$, et al. Regenerative medicine special feature: interrogating functional integration between injected pluripotent stem cell-derived cells and surrogate cardiac tissue [published online ahead of print October 21, 2009]. Proc Natl Acad Sci US A. doi:10.1073/pnas.0905729106.

35. Shin H, Zygourakis K, Farach-Carson MC, Yaszemski MJ, Mikos AG. Attachment, proliferation, and migration of marrow stromal osteoblasts cultured on biomimetic hydrogels modified with an osteopontin-derived peptide. Biomaterials. 2004;25(5):895-906.

36. Richardson TP, Peters MC, Ennett AB, Mooney DJ. Polymeric system for dual growth factor delivery. Nat Biotechnol. 2001;19(11):1029-1034.

37. Yang XB, et al. Human osteoprogenitor bone formation using encapsulated bone morphogenetic protein 2 in porous polymer scaffolds. Tissue Eng. 2004;10(7-8):1037-1045.

38. Jervis EJ, Guarna MM, Doheny JG, Haynes CA, Kilburn DG. Dynamic localization and persistent stimulation of factor-dependent cells by a stem cell factor / cellulose binding domain fusion protein. Biotechnol Bioeng. 2005;91(3):314-324.

39. Alberti K, et al. Functional immobilization of signaling proteins enables control of stem cell fate. Nat Methods. 2008;5(7):645-650.

40. Fan VH, et al. Tethered epidermal growth factor provides a survival advantage to mesenchymal stem cells. Stem Cells. 2007;25(5):1241-1251.

41. Suzuki T, et al. Highly efficient ex vivo expansion of human hematopoietic stem cells using Delta1-Fc chimeric protein. Stem Cells. 2006;24(11):2456-2465.

42. Kapur TA, Shoichet MS. Chemically-bound nerve growth factor for neural tissue engineering applications. J Biomater Sci Polym Ed. 2003;14(4):383-394.

43. Mapili G, Lu Y, Chen S, Roy K. Laser-layered microfabrication of spatially patterned functionalized tissue-engineering scaffolds. J Biomed Mater Res B Appl Biomater. 2005;75(2):414-424.

44. Mayer M, Yang J, Gitlin I, Gracias DH, Whitesides GM. Micropatterned agarose gels for stamping arrays of proteins and gradients of proteins. Proteomics. 2004;4(8):2366-2376.

45. Jiang X, Xu Q, Dertinger SK, Stroock AD, Fu TM, Whitesides GM. A general method for patterning gradients of biomolecules on surfaces using microfluidic networks. Anal Chem. 2005;77(8):2338-2347.

46. Nuttelman CR, Tripodi MC, Anseth KS. Dexamethasone-functionalized gels induce osteogenic differentiation of encapsulated hMSCs. J Biomed Mater Res A. 2006;76(1):183-195.

47. Kim S, Healy KE. Synthesis and characterization of injectable poly(N-isopropylacrylamide-co-acrylic acid) hydrogels with proteolytically degradable crosslinks. Biomacromolecules. 2003;4(5):1214-1223.

48. Li Jeon N, Baskaran H, Dertinger SK, Whitesides GM, Van de Water L, Toner M. Neutrophil chemotaxis in linear and complex gradients of interleukin- 8 formed in a microfabricated device. Nat Biotechnol. 2002;20(8):826-830. 
49. Kim L, Vahey MD, Lee HY, Voldman J. Microfluidic arrays for logarithmically perfused embryonic stem cell culture. Lab Chip. 2006;6(3):394-406.

50. Chung BG, et al. Human neural stem cell growth and differentiation in a gradient-generating microfluidic device. Lab Chip. 2005;5(4):401-406

51. Park TH, Shuler ML. Integration of cell culture and microfabrication technology. Biotechnol Prog. 2003;19(2):243-253.

52. Li YJ, Chung EH, Rodriguez RT, Firpo MT, Healy KE. Hydrogels as artificial matrices for human embryonic stem cell self-renewal. J Biomed Mater Res A. 2006;79(1):1-5

53. Biggs MJ, Richards RG, Gadegaard N, Wilkinson $\mathrm{CD}$, Oreffo RO, Dalby MJ. The use of nanoscale topography to modulate the dynamics of adhesion formation in primary osteoblasts and ERK/MAPK signalling in STRO-1+ enriched skeletal stem cells. Biomaterials. 2009;30(28):5094-5103.

54. Nelson CM, Jean RP, Tan JL, Liu WF, Sniadecki NJ, Spector AA, Chen CS. Emergent patterns of growth controlled by multicellular form and mechanics. Proc Natl Acad Sci U S A. 2005;102(33):11594-11599.

55. Anderson DG, Levenberg S, Langer R. Nanoliterscale synthesis of arrayed biomaterials and application to human embryonic stem cells. Nat Biotechnol. 2004;22(7):863-866.

56. Gerecht S, Burdick JA, Ferreira LS, Townsend SA, Langer R, Vunjak-Novakovic G. Hyaluronic acid hydrogel for controlled self-renewal and differentiation of human embryonic stem cells. Proc Natl Acad Sci U S A. 2007;104(27):11298-11303.

57. Oswald J, et al. Gene-expression profiling of CD34+ hematopoietic cells expanded in a collagen I matrix. Stem Cells. 2006;24(3):494-500.

58. Chua KN, Chai C, Lee PC, Ramakrishna S, Leong KW, Mao HQ. Functional nanofiber scaffolds with different spacers modulate adhesion and expansion of cryopreserved umbilical cord blood hematopoietic stem/progenitor cells. Exp Hematol. 2007;35(5):771-781.

59. Flaim CJ, Chien S, Bhatia SN. An extracellular matrix microarray for probing cellular differentiation. Nat Methods. 2005;2(2):119-125.

60. Taqvi S, Dixit L, Roy K. Biomaterial-based notch signaling for the differentiation of hematopoietic stem cells into T cells. J Biomed Mater Res A. 2006;79(3):689-697.

61. Awong G, Herer E, Surh CD, Dick JE, La MotteMohs RN, Zuniga-Pflucker JC. Characterization in vitro and engraftment potential in vivo of human progenitor $\mathrm{T}$ cells generated from hematopoietic stem cells. Blood. 2009;114(5):972-982

62. Nagaoka M, et al. Design of the artificial acellular feeder layer for the efficient propagation of mouse embryonic stem cells. J Biol Chem. 2008;283(39):26468-26476

63. Stevens MM, Mayer M, Anderson DG, Weibel DB, Whitesides GM, Langer R. Direct patterning of mammalian cells onto porous tissue engineering substrates using agarose stamps. Biomaterials. 2005;26(36):7636-7641.

64. Rosenthal A, Macdonald A, Voldman J. Cell patterning chip for controlling the stem cell microenvironment. Biomaterials. 2007;28(21):3208-3216.

65. Parekkadan B, et al. Cell-cell interaction modulates neuroectodermal specification of embryonic stem cells. Neurosci Lett. 2008;438(2):190-195.

66. Hui EE, Bhatia SN. Micromechanical control of cell-cell interactions. Proc Natl Acad Sci U S A. 2007;104(14):5722-5726.

67. Bhatia SN, Yarmush ML, Toner M. Controlling cell interactions by micropatterning in co-cultures: hepatocytes and $3 \mathrm{~T} 3$ fibroblasts.J Biomed Mater Res. 1997;34(2):189-199.

68. Fukuda J, et al. Micropatterned cell co-cultures using layer-by-layer deposition of extracell- ular matrix components. Biomaterials. 2006; 27(8):1479-1486.

69. Jinno S, et al. Microfabricated multilayer parylene-C stencils for the generation of patterned dynamic cocultures. J Biomed Mater Res A. 2008;86(1):278-288.

70. Guilak F, Cohen DM, Estes BT, Gimble JM, Liedtke W, Chen CS. Control of stem cell fate by physical interactions with the extracellular matrix. Cell Stem Cell. 2009;5(1):17-26.

71. Takeda H, Komori K, Nishikimi N, Nimura Y, Sokabe M, Naruse K. Bi-phasic activation of eNOS in response to uni-axial cyclic stretch is mediated by differential mechanisms in BAECs. Life Sci. 2006;79(3):233-239.

72. Tan JL, Tien J, Pirone DM, Gray DS, Bhadriraju K, Chen CS. Cells lying on a bed of microneedles: an approach to isolate mechanical force. Proc Natl Acad Sci US A. 2003;100(4):1484-1489.

73. Adamo L, et al. Biomechanical forces promote embryonic haematopoiesis. Nature. 2009; 459(7250):1131-1135.

74. North TE, et al. Hematopoietic stem cell development is dependent on blood flow. Cell. 2009; 137(4):736-748.

75. Engler AJ, Sen S, Sweeney HL, Discher DE. Matrix elasticity directs stem cell lineage specification. Cell. 2006;126(4):677-689.

76. McBeath R, Pirone DM, Nelson CM, Bhadriraju $\mathrm{K}$, Chen CS. Cell shape, cytoskeletal tension, and RhoA regulate stem cell lineage commitment. Dev Cell. 2004;6(4):483-495.

77. Tschumperlin DJ, et al. Mechanotransduction through growth-factor shedding into the extracellular space. Nature. 2004;429(6987):83-86.

78. Simon MC, Keith B. The role of oxygen availability in embryonic development and stem cell function. Nat Rev Mol Cell Biol. 2008;9(4):285-296.

79. Dang SM, Gerecht-Nir S, Chen J, Itskovitz-Eldor J, Zandstra PW. Controlled, scalable embryonic stem cell differentiation culture. Stem Cells. 2004;22(3):275-282.

80. Purpura KA, George SH, Dang SM, Choi K, Nagy A, Zandstra PW. Soluble FLt-1 regulates Flk-1 activation to control hematopoietic and endothelial development in an oxygen responsive manner. Stem Cells. 2008;26(11):2832-2842.

81. Hosokawa $\mathrm{K}$, et al. Function of oxidative stress in the regulation of hematopoietic stem cellniche interaction. Biochem Biophys Res Commun. 2007;363(3):578-583.

82. Park J, Bansal T, Pinelis M, Maharbiz MM. A microsystem for sensing and patterning oxidative microgradients during cell culture. Lab Chip. 2006;6(5):611-622.

83. Chaudhry MA, Vitalis TZ, Bowen BD, Piret JM. Basal medium composition and serum or serum replacement concentration influences on the maintenance of murine embryonic stem cells. Cytotechnology. 2008;58(3):173-179.

84. Krommenhoek EE, et al. Lab-scale fermentation tests of microchip with integrated electrochemical sensors for $\mathrm{pH}$, temperature, dissolved oxygen and viable biomass concentration. Biotechnol Bioeng. 2008;99(4):884-892.

85. Papin JA, Hunter T, Palsson BO, Subramaniam S. Reconstruction of cellular signalling networks and analysis of their properties. Nat Rev Mol Cell Biol. 2005;6(2):99-111

86. Han M, Gao X, Su JZ, Nie S. Quantum-dot-tagged microbeads for multiplexed optical coding of biomolecules. Nat Biotechnol. 2001;19(7):631-635.

87. Peerani R, Onishi K, Mahdavi A, Kumacheva E, Zandstra PW. Manipulation of signaling thresholds in "engineered stem cell niches" identifies design criteria for pluripotent stem cell screens. PLoS One. 2009;4(7):e6438.

88. Roeder I, Braesel K, Lorenz R, Loeffler M. Stem cell fate analysis revisited: interpretation of indi- vidual clone dynamics in the light of a new paradigm of stem cell organization.J Biomed Biotechnol. 2007;2007(3):84656.

89. Madlambayan GJ, et al. Dynamic changes in cellular and microenvironmental composition can be controlled to elicit in vitro human hematopoietic stem cell expansion. Exp Hematol. 2005;33(10):1229-1239.

90. Chen X, et al. Integration of external signaling pathways with the core transcriptional network in embryonic stem cells. Cell. 2008;133(6):1106-1117.

91. Bao S, et al. Epigenetic reversion of post-implantation epiblast to pluripotent embryonic stem cells. Nature. 2009;461(7268):1292-1295.

92. Lutolf MP, Hubbell JA. Synthetic biomaterials as instructive extracellular microenvironments for morphogenesis in tissue engineering. Nat Biotechnol. 2005;23(1):47-55.

93. Griffith LG, Swartz MA. Capturing complex 3D tissue physiology in vitro. Nat Rev Mol Cell Biol. 2006; 7(3):211-224

94. Hung PJ, Lee PJ, Sabounchi P, Lin R, Lee LP. Continuous perfusion microfluidic cell culture array for high-throughput cell-based assays. Biotechnol Bioeng. 2005;89(1):1-8.

95. DeForest CA, Polizzotti BD, Anseth KS. Sequential click reactions for synthesizing and patterning three-dimensional cell microenvironments. Nat Mater. 2009;8(8):659-664.

96. Kloxin AM, Kasko AM, Salinas CN, Anseth KS. Photodegradable hydrogels for dynamic tuning of physical and chemical properties. Science. 2009;324(5923):59-63.

97. Arai F, et al. Tie2/angiopoietin-1 signaling regulates hematopoietic stem cell quiescence in the bone marrow niche. Cell. 2004;118(2):149-161.

98. Matrosova VY, Orlovskaya IA, Serobyan N, Khaldoyanidi SK. Hyaluronic acid facilitates the recovery of hematopoiesis following 5-fluorouracil administration. Stem Cells. 2004;22(4):544-555.

99. Jin L, Hope KJ, Zhai Q, Smadja-Joffe F, Dick JE. Targeting of CD44 eradicates human acute myeloid leukemic stem cells. Nat Med. 2006;12(10):1167-1174.

100. Krause DS, Lazarides K, von Andrian UH, Van Etten RA. Requirement for CD44 in homing and engraftment of BCR-ABL-expressing leukemic stem cells. Nat Med. 2006;12(10):1175-1180.

101. Matsunaga $\mathrm{T}$, et al. Interaction between leukemiccell VLA-4 and stromal fibronectin is a decisive factor for minimal residual disease of acute myelogenous leukemia. Nat Med. 2003;9(9):1158-1165.

102.Liesveld JL, et al. Acute myelogenous leukemia-microenvironment interactions: role of endothelial cells and proteasome inhibition. Hematology. 2005; 10(6):483-494.

103.Nervi B, et al. Chemosensitization of acute myeloid leukemia (AML) following mobilization by the CXCR4 antagonist AMD3100. Blood. 2009;113(24):6206-6214.

104.Kaplan RN, Rafii S, Lyden D. Preparing the "soil": the premetastatic niche. Cancer Res. 2006; 66(23):11089-11093.

105. Kaplan RN, et al. VEGFR1-positive haematopoietic bone marrow progenitors initiate the pre-metastatic niche. Nature. 2005;438(7069):820-827.

106. Hiratsuka S, Watanabe A, Aburatani H, Maru Y. Tumour-mediated upregulation of chemoattractants and recruitment of myeloid cells predetermines lung metastasis. Nat Cell Biol. 2006; 8(12):1369-1375.

107. LaBarge MA, et al. Human mammary progenitor cell fate decisions are products of interactions with combinatorial microenvironments. Integr Biol. 2009;1:70-79.

108.LaBarge MA, Petersen OW, Bissell MJ. Of microenvironments and mammary stem cells. Stem Cell Rev. 2007;3(2):137-146. 
109.Levenberg S, Golub JS, Amit M, Itskovitz-Eldor J, Langer R. Endothelial cells derived from human embryonic stem cells. Proc Natl Acad Sci US A. 2002; 99(7):4391-4396.

110.Ungrin MD, Joshi C, Nica A, Bauwens C, Zandstra PW. Reproducible, ultra high-throughput formation of multicellular organization from single cell suspension-derived human embryonic stem cell aggregates. PLoS One. 2008;3(2):e1565.

111.Lee LH, Peerani R, Ungrin M, Joshi C, Kumacheva E, Zandstra P. Micropatterning of human embryonic stem cells dissects the mesoderm and endoderm lineages. Stem Cell Res. 2009;2(2):155-162.

112.Fok EY, Zandstra PW. Shear-controlled single-step mouse embryonic stem cell expansion and embryoid body-based differentiation. Stem Cells. 2005; 23(9):1333-1342

113. Bauwens C, Yin T, Dang S, Peerani R, Zandstra PW. Development of a perfusion fed bioreactor for embryonic stem cell-derived cardiomyocyte generation: oxygen-mediated enhancement of cardiomyocyte output. Biotechnol Bioeng. 2005; 90(4):452-461.

114.Purpura KA, George SH, Dang SM, Choi K, Nagy A, Zandstra PW. Soluble Flt-1 regulates Flk-1 activation to control hematopoietic and endothelial development in an oxygen-responsive manner. Stem Cells. 2008;26(11):2832-2842.

115.Yu TT, Shoichet MS. Guided cell adhesion and outgrowth in peptide-modified channels for neural tissue engineering. Biomaterials. 2005;
26(13):1507-1514.

116. Silva EA, Kim ES, Kong HJ, Mooney DJ. Material-based deployment enhances efficacy of endothelial progenitor cells. Proc Natl Acad Sci U S A. 2008;105(38):14347-14352

117. Sugiyama T, Kohara H, Noda M, Nagasawa T. Maintenance of the hematopoietic stem cell pool by CXCL12-CXCR4 chemokine signaling in bone marrow stromal cell niches. Immunity. 2006; 25(6):977-988.

118.Bhardwaj G, et al. Sonic hedgehog induces the proliferation of primitive human hematopoietic cells via BMP regulation. Nat Immunol. 2001; 2(2):172-180.

119. Kollet O, et al. Osteoclasts degrade endosteal components and promote mobilization of hematopoietic progenitor cells. Nat Med. 2006;12(6):657-664

120.Fleming HE, et al. Wnt signaling in the niche enforces hematopoietic stem cell quiescence and is necessary to preserve self-renewal in vivo. Cell Stem Cell. 2008;2(3):274-283.

121.Kopp HG, Avecilla ST, Hooper AT, Rafii S. The bone marrow vascular niche: home of HSC differentiation and mobilization. Physiology (Bethesda). 2005;20:349-356.

122.Adams GB, et al. Stem cell engraftment at the endosteal niche is specified by the calcium-sensing receptor. Nature. 2006;439(7076):599-603.

123. Papayannopoulou T. Current mechanistic scenarios in hematopoietic stem/progenitor cell mobilization. Blood. 2004;103(5):1580-1585.
124. Nilsson SK, et al. Osteopontin, a key component of the hematopoietic stem cell niche and regulator of primitive hematopoietic progenitor cells. Blood. 2005;106(4):1232-1239.

125. Craddock CF, Nakamoto B, Andrews RG, Priestley GV, Papayannopoulou T. Antibodies to VLA4 integrin mobilize long-term repopulating cells and augment cytokine-induced mobilization in primates and mice. Blood. 1997;90(12):4779-4788.

126.Pruijt JF, van Kooyk Y, Figdor CG, Lindley IJ, Willemze R, Fibbe WE. Anti-LFA-1 blocking antibodies prevent mobilization of hematopoietic progenitor cells induced by interleukin-8. Blood. 1998;91(11):4099-4105.

127. Jung Y, et al. Annexin II expressed by osteoblasts and endothelial cells regulates stem cell adhesion, homing, and engraftment following transplantation. Blood. 2007;110(1):82-90

128. Weber JM, Calvi LM. Notch signaling and the bone marrow hematopoietic stem cell niche [published online ahead of print August 11, 2009]. Bone. doi:10.1016/j.bone.2009.08.007.

129. Kurth I, Franke K, Pompe T, Bornhauser M, Werner C. Hematopoietic stem and progenitor cells in adhesive microcavities. Integr Biol. 2009;1:427-434.

130.Walker MR, Patel KK, Stappenbeck TS. The stem cell niche. J Pathol. 2009;217(2):169-180.

131.Mendez-Ferrer S, Lucas D, Battista M, Frenette PS. Haematopoietic stem cell release is regulated by circadian oscillations. Nature. 2008;452(7186):442-447. 